In dieser Rubrik referieren und kommentieren diese Experten für Sie Arbeiten aus der internationalen Fachliteratur.

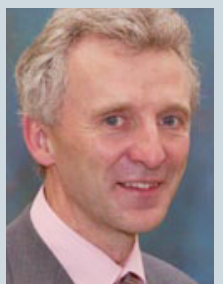

Dr. med.

Martin

Claßen,

Bremen

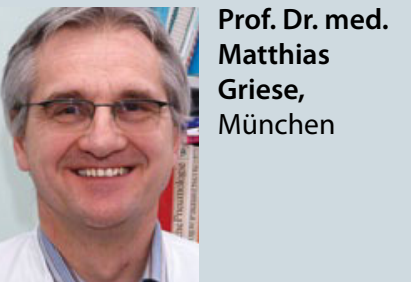

Prof. Dr. med. Gerd Horneff, Sankt Augustin

\section{Unnötiger Antibiotikagebrauch begünstigt eine NEC}

In einer Studie von der Yale University School of Medicine fanden Wissenschaftler heraus, dass die Dauer einer Antibiotikagabe bei Frühgeborenen ohne Sepsis mit einem erhöhten Risiko für eine NEC assoziiert ist.

$\mathrm{D}$ ie nekrotisierende Enterokolitis (NEC) stellt auch in unseren Tagen eine bedrohliche (Mortalität 25-33\%) und häufige Komplikation der Frühgeburtlichkeit dar. 1-5\% der Kinder unter $1.500 \mathrm{~g}$ sind betroffen. Neben der Unreife des Darms und des Immunsystems spielt die bakterielle Kolonisation des Gastrointestinaltraktes eine relevante Rolle. Präventiv wirken u. a. Probiotikamischungen.
Den Einfluss von Länge und Indikation antibiotischer Therapien bei Frühgeborenen auf die NEC-Inzidenz wurde von einer amerikanischen Arbeitsgruppe untersucht. 124 Kinder mit manifester NEC wurden in einer 1:2 Fall-Kontroll-Studie mit 248 gematchten Fällen verglichen.

Eine Vielzahl von Risikofaktoren (u. a. Respiratory Distress Syndrome [RDS], Small for Gestational Age, persistierender Ductus arteriosus [PDA], Sepsis,

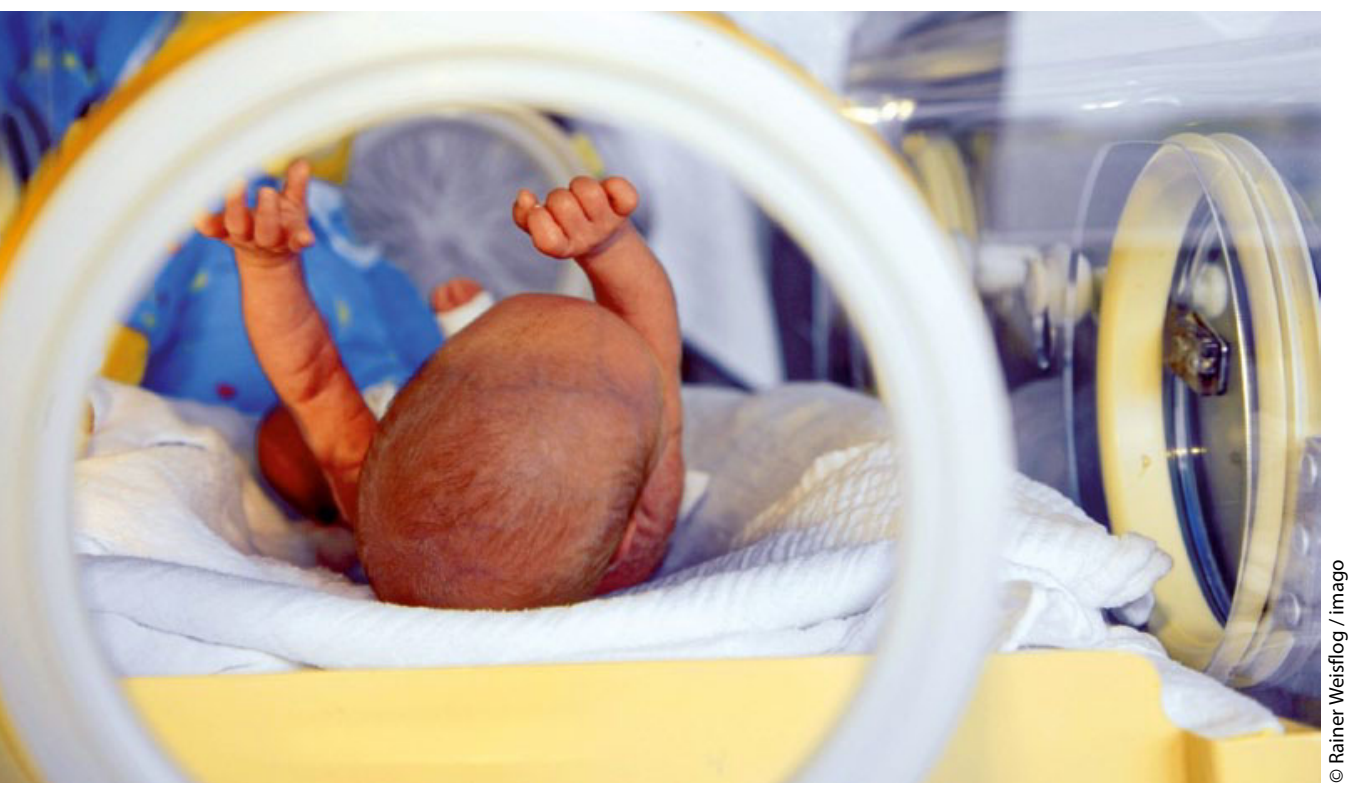

Die Indikation und Länge einer antibiotischen Therapien sollten auch bei Frühchen gut abgewogen werden.
Nahrungsaufbau) wurde erfasst und analysiert. Kinder mit RDS oder PDA hatten ein geringeres NEC-Risiko. In der NECGruppe fanden sich erwartungsgemäß deutlich mehr Kinder mit Bakteriennachweis in der Blutkultur bzw. Sepsis. Die multivariate Analyse aller Kinder ohne Sepsis, die antibiotisch behandelt worden waren, ohne dass eine Bakterämie nachgewiesen wurde, ergab folgendes Ergebnis: Mit jedem Tag der (unnötigen) Antibiotikatherapie stieg das NEC-Risiko bis auf eine Odds Ratio von 2,94 nach zehn Tagen Antibiotikatherapie.

Alexander $\mathrm{VN}$ et al. Antibiotic exposure in the newborn intensive care unit and the risk of necrotizing enterocolitis. J Pediatr 2011; 159: 392-7

Kommentar: Die Entstehung einer NEC ist von vielen Variablen abhängig, die auch nur teilweise beeinflussbar sind. Die Faktoren, die beeinflussbar sind, müssen im Sinne einer Risikominimierung beachtet werden. Neben der Vermeidung unnötiger Bluttransfusionen [vgl. pädiatrie hautnah 2011; 2: 222] gehört dazu u. a. eine probiotische Therapie. Wie die Studie zeigt, muss die Vermeidung oder Abkürzung antibiotischer Therapien ebenfalls bedacht werden - selbstverständlich immer unter Risikoabwägung, denn eine Sepsis gehört auch in der zitierten Untersuchung zu den wichtigsten Risikofaktoren für eine NEC. Die Überprüfung von Indikation und Länge antibiotischer Therapien auch bei Frühgeborenen passt darüber hinaus gut zur aktuellen Mediendiskussion über Hygieneprobleme und multiresistente Keime auf neonatologischen Intensivstationen.

Dr. Martin Claßen 